\title{
Cardiovascular Impacts of COVID-19 Pandemic: From Presentation to Management: Current and Future Perspectives
}

\author{
Zainab Atiyah Dakhil ${ }^{1^{*}}$, Hasan Ali Farhan ${ }^{2,3}$
}

\footnotetext{
${ }^{1}$ AL-Kindy College of Medicine, Baghdad University, Baghdad, Iraq ${ }^{2}$ Scientific Council of Cardiology, Iraqi Board for Medical Specializations, Baghdad, Iraq ${ }^{3}$ Baghdad Heart Centre/Medical City, Baghdad, Iraq
}

\begin{abstract}
Corona virus disease 2019 (COVID-19) pandemic represents a global unprecedented healthcare crisis that results in respiratory syndrome and can cause remarkable cardiovascular impacts in form of myocardial injury, myocarditis, heart failure and arrhythmia. This review aimed to provide clinical landscape of this novel virus in the context of cardiovascular system involvement, in addition to explore future perspectives regarding response of healthcare system to this outbreak. The study showed that elderly patients and those with comorbidities are most susceptible for this infection, and will have the worst prognosis. Poor prognostic determinants for this disease were higher C-reactive protein, IL-6, ferritin, serum troponin and NT-ProBNP. Considering prominent role of COVID19 pandemic on cardiovascular health and care on multiple levels there is no time better than now for cardiology community to play a key role in fighting this pandemic globally in form of solidarity with other specialities and accelerating knowledge about this health threat in addition to adopt new perspectives on learning and training to face this unprecedented crisis.
\end{abstract}

Keywords: public health, pandemic, biomarkers, myocarditis, troponin

\section{Correspondence:}

Zainab Atiyah Dakhil

Address: AL-Kindy College of Medicine/ Baghdad University, Iraq

Email: stethoscope.med@gmail.com
Received: 09.04.2020,

Accepted: 10.04 .2020

https://doi.org/10.5799/jcei/7941

\section{INTRODUCTION}

In December 2019 in China, in Wuhan town, an outbreak of virus caused respiratory infection that ranges from asymptomatic infection to severe viral pneumonia causing respiratory failure, which was called later by WHO as COVID19 (Corona Virus 2019) [1,2]. The high infectivity along with ability to be transmitted during asymptomatic period resulted in rapid transmission of the virus to many countries [3] to be considered on March 11, 2020 by WHO as a pandemic [4]. COVID-19 has been diagnosed in $\mathbf{1 , 5 9 2 , 8 0 1}$ cases around the world and caused death in 95,021 patients, last updated; 2020 April 9 (21:11 GMT) [5], the numbers are just increasing enormously. As the most common organ to be affected by COVID-19 infection after lung is heart [6], so our review aimed to provide a demographic, clinical and investigational landscapes of this novel virus in context of cardiovascular system involvement, in addition to explore the present and future perspectives of this global pandemic on cardiovascular care.

\section{METHODS}

A Search method of PubMed and google search engines was conducted to find articles published since start of COVID-19 outbreak. Search included original researches, review articles, consensus statements and guidelines, expert viewpoints and editorials; all were included. Search words used were: "COVID-19" or "Coronavirus" or "SARS-COV2" and "cardiovascular" or "cardiac" or "heart failure" or myocarditis" or "pericarditis" or "myocardial infarction" or "troponin."

\section{SO FAR, WHAT DO WE KNOW?}

\section{Demographic Characteristics}

Zou et al. reported in his cohort study of COVID-19 patients that they were more to be males $62 \%$ with a mean age of 56 years, $50 \%$ of patients had comorbidities prior to infection, most commonly hypertension $30 \%$, diabetes $19 \%$, coronary heart disease 


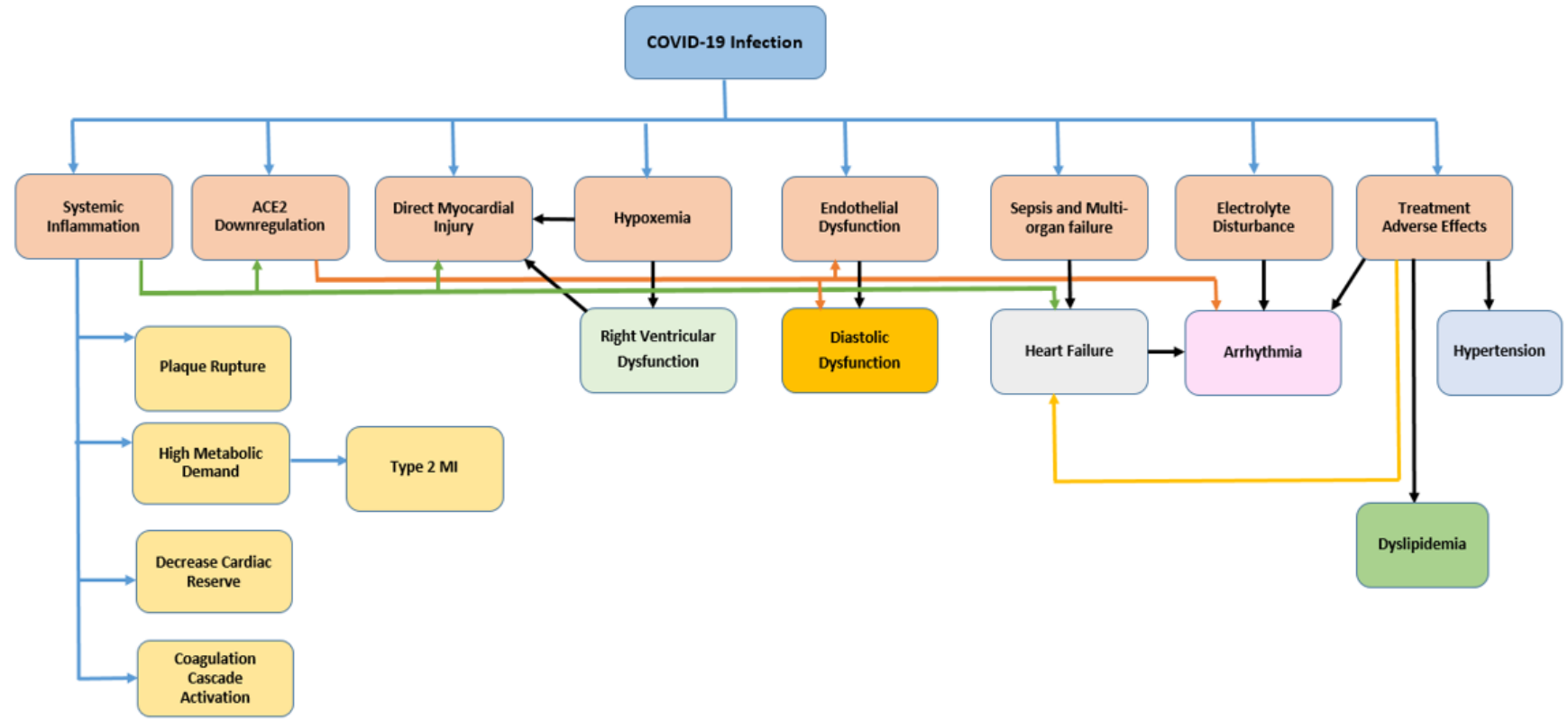

Figure 1. Mechanisms of Cardiovascular Effects of COVID-19

(CHD) $8 \%$ and chronic obstructive pulmonary disease (COPD) in 3\% [7], while Guo et al suggested in his study that COVID-19 patients were more to be females $51.3 \%$ with a mean age of 58.5 years, and that hypertension and diabetes were most common comorbidities in $32.6 \%$ and $15 \%$ respectively, in the time that $11.2 \%$ and $4.3 \%$ had prior history of CHD and cardiomyopathy (CMP) respectively [8]. Arentz et al described the main demographics of COVID-19 patients who were critically ill and admitted to intensive care unit (ICU) and showed that they were males in 52\% and mean age was 70 years, critically ill patients cohort had more comorbidities 86\%; with chronic kidney disease (CKD) and heart failure (HF) were the most common comorbidities in those patients $47.6 \%$ and $42.9 \%$ respectively and diabetes and COPD were evident in 33.3\% for each [9] while Graziano et al revealed that critically ill patients were more to be males and the mean age was 79.5 years, main comorbidity is CHD that reported in $35.5 \%$ followed by diabetes $20.3 \%$ [10]. Patients who had myocardial injury (MI) in view of COVID19 infection were of mean age of 78.5 years, $50 \%$ were males, $66.67 \%$ of them were hypertensive, cardiovascular disease was reported in $66.7 \%$, COPD and hypertension were evident in history in $33.33 \%$ for each. Interestingly those with MI had more than two comorbidities in $83.33 \%$ of patients [11].

\section{Cardiovascular Manifestations}

Ding et al. revealed that the earlier SARS-COV had not shown significant distribution in heart, unlike lung and kidneys, however other researchers suggested that the heart was associated with greater myocardial infiltration and myocardial damage in SARS-COV [12-17]. SARS-COV2 has remarkable distribution in heart tissue in 35\% of autopsies and in up to $42.1 \%$ of patients with heart failure in COVID19 infection $[11,15]$. This can explain the dominance of heart involvement just next to lung in this pandemic as cardiac tissue can be damaged directly by virus infiltration $[11,15]$. COVID-19 infection can be caused by binding of virus to ACE2 receptor which mainly expressed in heart and lung resulting in ACE2 downregulation which cause respiratory and cardiac manifestations in form of myocardial inflammation, lung oedema in addition to respiratory failure, furthermore, ACE2 downregulation can cause endothelial dysfunction which explains the diastolic dysfunction that reported in patients with COVID-19 $[15,18]$. Cytokine storm had been evident in COVID-19 which can have a vital role in progression of inflammation and contributes to decrease coronary blood flow and oxygen supply, inflammation can also induce rupture of coronary plaques and coagulation cascade activation with increase thrombosis risk, in addition to COVID-19 effect in increasing metabolic demands and decrease cardiovascular reserve $[1,19,20]$. Cardiovascular impacts of COVID-19 infection are demonstrated in Figure 1. Whether or not long-term cardiovascular complications are possible cannot be determined now and need further future studies.

\section{Myocardial injury}

Myocardial injury is defined as increase in levels of troponin $\mathrm{T}(\mathrm{TnT})$ more than $99^{\text {th }}$ percentile upper reference limit [21]. Most researches in context of novel COVID-19 depend on this definition. Xu et al recorded MI in 11.3\% of COVID-19 cohorts while Huang et al and Wang et al reported $\mathrm{MI}$ in $12 \%$ and $7.2 \%$ of COVID-19 patients $[11,19,22]$. MI can be explained by systemic inflammation, increasing demand and hypoxia. Main risk factors for developing $\mathrm{MI}$ were reported by $\mathrm{Xu}$ et al as older age, increasing CRP and novel corona virus pneumonia (NCP) severity due to increase inflammatory status of those patients, also presence of prior history of $\mathrm{CHD}$ was reported 
in all COVID-19 patients who had MI [11]. Furthermore, Guo et al suggested hypertension, CHD, CMP, Diabetes, COPD and CKD more in patients with MI [8]. Patients with myocardial injury were more susceptible for hypoxaemia, NCP and with increased need for ICU admission, moreover patients with high troponin were at more risk to develop acute kidney injury (AKI) which occurred in all patients with MI, 41.67\% with high troponin but not fulfilled definition of MI [11].

\section{Myocarditis}

Myocarditis can be caused by variable viruses and manifested as severe rapidly deteriorating LV dysfunction and cardiogenic shock with elevated cardiac enzymes, mortality rate can reach 50-70\% [23]. Fulminant myocarditis was reported in COVID-19 [6]. It was suggested that immune injury can be the cause of fulminant myocarditis which either occurs early or never in context of COVID-19, and the prognosis can be better if no malignant arrhythmias complicated the course of illness [6]. Myocarditis is known to be caused by other viruses that caused respiratory syndromes (SARS, MERS) [12-14,16,17]. Earlier researches in MERS-CoV have revealed myocarditis and damage were evident by cardiac MRI (CMR) [17]. Death was attributed in $7 \%$ to myocarditis with circulatory failure and in $33 \%$ of cases myocarditis contributed to the patient's death [24]. However, CMR could not be performed in any of COVID19 cohorts due to infection control restrictions.

\section{Heart failure}

Heart failure was reported in $37.7 \%$ of COVID-19 patients [11]. Heart failure can be explained by the fore mentioned mechanisms. Arentz et al. revealed that CMP occur in $33.3 \%$ of critically ill COVID-19 patients, pulmonary oedema was recorded in $9.5 \%$ of total patients and in $42.9 \%$ of those with preadmission heart failure, this high percentage can be part of COVID-19 infection or due multisystem failure or due to high number of patients with baseline cardiovascular disease [9] .Heart failure was an important predictor of mortality in COVID-19, as it was recorded in 52\% of COVID-19 non survivors and in $12 \%$ of those who recovered [22].

\section{Arrhythmia}

Arrhythmias can occur in COVID-19 as part of myocardial injury, myocarditis, cardiac dysfunction or due to electrolyte disturbance that especially occur in critically ill patients in addition to being part of adverse effects of drugs used in treating this population [3,25]. Wang et al reported arrhythmia in $16.7 \%$ of total COVID-19 patients, arrhythmia was reported more in those who required ICU admission $44.4 \%$ versus $8.9 \%$ in those who did not [22].

\section{Shock}

Cardiogenic, septic or mixed cardiac plus primary pulmonary causes of shock or respiratory complaints in COVID-19 are important to be considered. Due to limitation in using right heart catheterization it could be difficult to differentiate between different types of shock in a COVID19 patients [27]. However, bedside echo study and biomarkers like Pro-BNP and inflammatory markers can give a clue to type of shock. Wang et al in his study reported cardiogenic shock $8.7 \%$ of patients, which was higher significantly in those who were admitted to ICU $30.6 \%$ versus who were not $2 \%$ [22].

\section{Pericardial disease}

Pericardial involvement was reported in COVID-19 patients, a case of myopericarditis with elevated markers of myocardial necrosis was described in a COVID-19 patient [27]. Furthermore, Hua et al reported first case of cardiac tamponade in a COVID-19 case [28].

\section{Investigational Parameters in COVID-19}

There is remarkable knowledge gap regarding main haematological and biochemical parameters in in COVID19 infection, however, there are several studies that gave clues regarding this issue. Lymphopenia and leucocytosis were reported in prior cohorts of COVID-19 [7,11]. Moreover, it was suggested that the severity of lymphopenia reflects extent of myocardial damage as it was reported in $83.33 \%$ of patients with COVID-19 and myocardial injury versus $37.5 \%$ and $34.78 \%$ of those with elevated troponin and those with normal troponin levels respectively [11]. Haemoglobin and platelet count were lower in COVID-19 patients who had myocardial injury [11]. Biochemical parameters correlated with extent of myocardial injury, as creatinine, alanine aminotransferase, triglycerides and lactic acid were higher in patients with high troponin $\mathrm{T}$ levels, while total cholesterol, HDL, LDL and aspartate aminotransferase did not differ significantly between those with high troponin $\mathrm{T}$ versus normal troponin [8]. Moreover, it had been suggested that there is positive correlation between severity of respiratory disease in COVID-19 and lactate dehydrogenase, serum amyloid $\mathrm{A}$ and C-reactive protein (CRP), while there is negative correlation between respiratory disease severity and lymphocyte counts, albumin, serum potassium and HDL, serum potassium was considered as a good prognostic indicator as continuous renal potassium loss can result from degradation of ACE2 in COVID-19 infection [29].

Cardiac enzymes like creatinine kinase, myoglobin significantly elevated in COVID-19 patients [8]. Cardiac troponin I was only marginally elevated in COVID-19 infection where by myocardial injury was defined in $8-12 \%$ and it was mainly caused by cytokine storm [30,31]. Troponin $\mathrm{T}$ had further prognostic impact in that high troponin $\mathrm{T}$ patients had more complications including ARDS, VT/VF, acute coagulopathy and AKI, additionally, high troponin $\mathrm{T}$ levels in patients with CVD had the highest mortality and shortest survival term [8]. High sensitivity troponin I during hospitalization found in 50\% of nonsurvivors COVID-19, it increased rapidly from day 16 after disease onset [7]. However, most COVID-19 cohorts did not 
Table 1. Cardiovascular Adverse Effects Related to Drugs Used in COVID-19

\begin{tabular}{ll}
\hline Cardiovascular Adverse Effects & Causative Drugs \\
\hline Long QT/Torsades de pointes & chloroquine, azithromycin, lopinavir, ritonavir, tocilizumab \\
\hline Dyslipidaemia & Lopinavir, Ritonavir, steroids, tocilizumab \\
\hline Metabolic Syndrome & Lopinavir, Ritonavir, Steroids \\
\hline Conduction abnormalities & Chloroquine, lopinavir, ritonavir \\
\hline Atrial fibrillation/flutter/VT & steroids \\
\hline Hypertension & steroids \\
\hline Fluid retention & steroids \\
\hline Cardiomyopathy (dilated/restrictive) & Chloroquine \\
\hline
\end{tabular}

assess the timing of troponin samples, troponin also can be elevated due to non-cardiac causes in context of critical illness.

Chen Chen et al. reported increased NT-ProBNP in 27.5\% of COVID-19 patients [30]. Moreover, Guo et al reported remarkable increase with escalation in NT-ProBNP levels in COVID-19 non survivors than survivors, additionally, there was increasing levels of NT-ProBNP correlated with increasing level of troponin $\mathrm{T}$ and with higher incidence with malignant arrhythmias and poorer outcomes [8]. D-dimer was detected in context of abnormal coagulation parameters in severe COVID-19 patients and it was associated with increasing cardiac troponin and it signified higher mortality [7,8,11,32]. Procalcitonin associated with increasing severity of disease and it suggested multiple infections in critically ill patients and associated with worse prognosis [29]. High serum ferritin, IL-6 and CRP detected in COVID-19 patients [7] and indicate implicated inflammation in pathogenesis of COVID-19. Additionally, they can serve as poor prognostic indicators [7].

\section{Electrocardiographic and Echocardiographic Changes}

$\mathrm{Xu}$ et al. reported that ECG abnormalities were evident in all COVID-19 patients with myocardial injury versus $20.38 \%$ of those with high troponin not fulfilling MI definition. Atrioventricular block, arrhythmias, ST/T changes and Q waves also were reported. None of patients with normal cardiac biomarker had ECG abnormalities [11]. Diffuse ST elevation was reported in a COVID-19 case with elevated cardiac enzymes who was subsequently diagnosed as myopericarditis after undergoing coronary angiography that showed non-obstructive coronaries [27]. S1Q3T3, ST elevation and RBBB all were reported in patients with COVID-19 [33].

Echocardiographic changes were recorded in COVID-19 patients in $45.28 \%$, most frequent in patients with MI (100\%) versus (13.04\%) of those with normal cardiac biomarkers [11]. Left ventricular thickening and left ventricular dilatation were most common findings; $12.5 \%$ for each, other reported findings were left atrial enlargement, mitral regurgitation, tricuspid regurgitation and aortic regurgitation [11]. Myocardial wall thickening was reported in patients with COVID-19 mainly due to cytokine storm which causes increased vascular permeability and myocardial oedema [6].

However, ECG and echocardiographic changes that reported in COVID-19 cannot be only implicated due to infection itself, as large percentage of the infected patients had baseline hypertension and cardiovascular diseases that can cause such changes.

\section{COVID-19 Therapy Impacts on Cardiovascular}

\section{System}

Cardiovascular Effects of drugs used in treatment of COVID-19 infection [26,34-37] are summarised in Table 1.

\section{CURRENT AND FUTURE PERSPECTIVES}

Most conducted COVID-19 researches emphasized on vulnerability of patients with baseline CVD to acquire COVID-19 infections and their worse prognosis when compared to those with no CVD, which is not the only thing that raises our concern regarding cardiovascular care (CVC) but the impact of changing healthcare protocols coping with recent pandemic which can impact CVC for other Non COVID-19 patients. Despite recent SCAI guidelines recommending all STEMI patients should be sent for catheterization, use of thrombolytic therapy has been justified if local resources were overwhelmed or there is high COVID-19 prevalence especially in low risk STEMI patients (inferior STEMI with no right ventricular involvement or in lateral STEMI with no haemodynamic compromise) [38]. It had been suggested that number of patients with STEMI who sought medical help was little compared to prior to pandemic in addition to delay in asking for help if done, as patients were reluctant to attend hospitals in view of strict infection control strategies, additionally timing to reperfusion in those patients was longer than prior to pandemic which can be explained by detailed history and travel history taking, performing investigations like CXR and PCR and making the patient wear protection equipment prior to intervention all that can contribute to delay [39]. Furthermore, poor access to hospitals, lack of transportation and already burdened facilities, all can delay managing those patients, which subsequently will have devastating impacts 
on outcomes. Whether adopting universal conservative strategy in STEMI and high risk NSTEMI will impact the long-term cardiovascular prognosis worse than COVID-19 itself warranted further studies in future. Up to $20 \%$ of COVID-19 patients in China were hospitalized and 5\% required ICU admission, while in Italy up to $40 \%$ of patients were hospitalized and $7 \%$ were ICU admitted [40,41] this signifies the importance of healthcare system dealing with COVID-19 challenges. WHO had issued many steps to be adopted by healthcare systems as part of COVID-19 response including but not limited to surveillance, infection prevention and control and rapid case investigation and management, additionally, WHO suggested to maintain continuity of essential services while freeing up capacity for COVID-19 cases in addition to review supply chains and equipment of health technologies [42].

Frontline doctors and medical staff are the key players to fight this challenging pandemic, their safety and ensured wellbeing are the most vital in confronting COVID-19. It is crucial to train and repurpose the healthcare providers according to need of priority [42], in addition to preparing a well-equipped workforce nucleus amidst the crisis with full training especially regarding putting on and off personal protection equipment (PPE), dealing with suspected and infected patients, performing intubation, cardiopulmonary resuscitation and training on dealing with ventilators according to pandemic protocols [38].

Prior global spread of viral infections like SARS rang a bell of danger back then and called for actions by healthcare systems and policymakers to prepare systems to face the anticipated challenges. During earlier SARS infection there was remarkable shortage in PPE, shortage of isolation facilities [43], same as current pandemic in addition to under-preparation and training of healthcare facilities and healthcare providers to confront such pandemics despite prior experiences.

It is important to consider in future establishing advanced infection control protocols, proper isolation ICU, cardiac care unit (CCU), catheterization labs and recovery beds in order not only to be able to help COVID-19 patients who are at high risk of CVD but also to continue providing emergency interventions as necessary to general population without breaching infection prevention and control protocols.

Policy makers and medical educationalists all should adopt new future perspective in preparing a well-equipped healthcare system that not only being patient centred but also public centred in providing healthcare with establishing framed roadmaps to follow during such massive crisis without crashing the healthcare economics or compromising essential medical services for general population.

\section{CURRENT AND FUTURE DIRETIONS}

This study revealed the prominent role of COVID19 pandemic on cardiovascular health and care on multiple levels with long-term cardiovascular impact of this pandemic warranted further studies, there is no time better than now for cardiology community to play a key role in fighting this pandemic globally in form of solidarity with other specialities and accelerating knowledge about this health threat in addition to adopt a new perspective on learning and training to face this unprecedented crisis. Every effort should be made to adopt future healthcare viewpoints that become public centred to overcome such crisis without compromising vital general medical services and healthcare economics.

\section{ACKNOWLEDGEMENTS}

The authors thank all frontline doctors and medical staff who are confronting this pandemic with extraordinary courage, commitment and patience and all healthcare providers who sacrificed their lives while saving their patients.

Declaration of interest: The authors report no conflicts of interest.

Financial Disclosure: No financial support was received.

\section{REFERENCES}

1. Huang C, Wang $\mathrm{Y}, \mathrm{Li} \mathrm{X}$, et al. Clinical features of patients infected with 2019 novel coronavirus in Wuhan, China. Lancet 2020;395:497-506. doi: 10.1016/S0140-67 36(20)30183-5.

2. Chen N, Zhou M, Dong X, et al. Epidemiological and clinical characteristics of 99 cases of 2019 novel coronavirus pneumonia in Wuhan, China: a descriptive study. Lancet 020;395:507-13. doi: 10.1016/S0140-6736 (20)30211-7.

3. Bansal M. Cardiovascular disease and COVID-19. Diabetes \& Metabolic Syndrome: Clinical Research \& Reviews. 2020. doi: 10.1016/j.dsx.2020.03.013.

4. World Health Organization [Internet]. WHO announces COVID-19 outbreak a pandemic;2020 March 11 [Cited March 29]. Available at: http://www. euro.who.int/en/health-topics/health-emergencies/coro navirus-covid-19/news/news/2020/3/who-announces-c ovid-19-outbreak-a-pandemic (Accessed April 1, 2020).

5. Worldmeters.info. [Internet]. COVID-19 Corona Virus Pandemic; 2020 April 9 [Cited 2020 April 9 (21:11 GMT)]. Available at: https://www.worldometers.info/ coronavirus/?utm_campaign=homeAdvegas 1 ?

6. Zeng JH, Liu Y-X, et al. First Case of COVID-19 Infection with Fulminant Myocarditis Complication: Case Report and Insights. Preprints 2020, 2020030180 doi: 10.20944/preprints202003.0180.v1.

7. Zhou F, Yu T, Du R, et al. Clinical course and risk factors for mortality of adult inpatients with COVID-19 in Wuhan, China: a retrospective cohort study. The Lancet 2020. doi: 10.1016/S0140-6736(20)30566-3. 
8. Guo T, Fan Y, Chen M, et al. Cardiovascular implications of fatal outcomes of patients with coronavirus disease 2019 (COVID-19). JAMA cardiology. 2020 Mar 27. doi: 10.1001/jamacardio. 2020.1017.

9. Arentz M, Yim E, et al. Characteristics and outcomes of 21 critically ill patients with COVID-19 in Washington State. JAMA 2020. doi: 10.1001/jama.2020.4326.

10. Onder G, Rezza G, Brusaferro S. Case-fatality rate and characteristics of patients dying in relation to COVID19 in Italy. JAMA 2020. doi: 10.1001/jama.2020.4683.

11. $\mathrm{Xu} \mathrm{H}, \mathrm{Hou} \mathrm{K}$, et al. Acute Myocardial Injury of Patients with Coronavirus Disease 2019. medRxiv (2020). doi: 10.1101/2020.03.05.20031591.

12. Assiri A, Al-Tawfiq JA, Al-Rabeeah AA, et al. Epidemiological, demographic, and clinical characteristics of 47 cases of Middle East respiratory syndrome coronavirus disease from Saudi Arabia: a descriptive study. Lancet Infect Dis. 2013;13(9):752-61. doi: 10.1016/S1473-3099(13)70204-4.

13. Edwards S, Small JD, Geratz JD, Alexander LK, Baric RS. An experimental model for myocarditis and congestive heart failure after rabbit coronavirus infection. J Infect Dis. 1992;165(1):134-40. doi: 10.1093/infdis/165.1.134.

14. Ding Y, Li H, Zhang Q, et al. Organ distribution of severe acute respiratory syndrome (SARS) associated coronavirus (SARS-CoV) in SARS patients: implications for pathogenesis and virus transmission pathways. The Journal of Pathology: A Journal of the Pathological Society of Great Britain and Ireland 2004;203(2):622-30. PMID: 15141376, doi: 10.1002/path.1560.

15. Oudit GY, Z Kassiri, et al. SARS-coronavirus modulation of myocardial ACE2 expression and inflammation in patients with SARS. European journal of clinical investigation 2009;39(7):618-25. PMID: 19453650, doi: 10.1111/j.1365-2362.2009.02153.x

16. Han Y, Geng H, Feng W, Tang X, Ou A, Lao Y, et al. A follow-up study of 69 discharged SARS patients. J Tradit Chin Med. 2003;23(3):214-7. PMID: 14535196.

17. Alhogbani T. Acute myocarditis associated with novel Middle east respiratory syndrome coronavirus. Ann Saudi Med. 2016;36(1):78-80. PMID: 26922692, doi: 10.5144/0256-4947.2016.78.

18. Guo J, Huang Z, Lin L, Jiagao L. Coronavirus Disease 2019 (COVID-19) and Cardiovascular Disease: A Viewpoint on the Potential Influence of AngiotensinConverting Enzyme Inhibitors/Angiotensin Receptor Blockers on Onset and Severity of Severe Acute Respiratory Syndrome Coronavirus 2 Infection. Journal of the American Heart Association. 2020;9:e16219. doi: 10.1161/JAHA.120.016219

19. Xiong T-Y, Redwood S, Prendergast B, Chen M. Coronaviruses and the cardiovascular system: acute and long-term implications. European Heart Journal 2020. doi: 10.1093/eurheartj/ehaa231.
20. Libby P, Simon DI. Inflammation and thrombosis: the clot thickens. Circulation 2001:1718-20. PMID: 22785436.

21. Thygesen K, Alpert JS, Jaffe AS, et al. Fourth Universal Definition of Myocardial Infarction. J Am Coll Cardiol 2018;72:2231-64. doi: 10.1161/CIR.0000000000000617.

22. Wang $\mathrm{D}, \mathrm{Hu} \mathrm{B}$, et al. Clinical characteristics of 138 hospitalized patients with 2019 novel coronavirusinfected pneumonia in Wuhan, China. JAMA 2020. doi: 10.1001/jama.2020.1585.

23. Wang D, Li S, Jiang J, et al. Chinese society of cardiology expert consensus statement on the diagnosis and treatment of adult fulminant myocarditis. Science China Life sciences. 2019;62(2):187-202. doi: 10.1007/s11427018-9385-3.

24. Ruan Q, Yang K, Wang W, Jiang L, Song J. Clinical predictors of mortality due to COVID-19based on an analysis of data of 150 patients from Wuhan, China. Intensive Care Med 2020. PMID: 32125452, doi: 10.1007/s00134-020-05991-x .

25. Elsevier.com. [Internet]. Tim Smith and Tony Prosser. Clinical Drug Information-Clinical Solutions. Available at https://www.elsevier.com/_data/assets/pdf_file/ 0007/988648/COVID-19-Drug-Therapy_Mar-2020.pdf

26. Driggin E, Madhavan MV, Bikdeli B, et al. Cardiovascular Considerations for Patients, Health Care Workers, and Health Systems During the Coronavirus Disease 2019 (COVID-19) Pandemic, Journal of the American College of Cardiology 2020. doi: 10.1016/j.jacc.2020.03.031.

27. Inciardi, Riccardo M., Laura Lupi, et al. Cardiac Involvement in a Patient with Coronavirus Disease 2019 (COVID-19). JAMA cardiology 2020. doi: 10.1001/jamacardio.2020.1096.

28. Hua A, O’Gallagher K, Sado D, Byrne J. Life-threatening cardiac tamponade complicating myo-pericarditis in COVID-19. European Heart Journal 2020. doi: 10.1093/eurheartj/ehaa253.

29. Wang D, Li R-F, Wang J, et al. Association between severity of COVID-19 and clinical and biochemical characteristics: a cross-sectional study, 24 March 2020, Preprint (Version 1) available at Research Square doi: 10.21203/rs.3.rs-18482/v1.

30. Chen C, Zhou Y, Wang DW. SARS-CoV-2: a potential novel etiology of fulminant myocarditis. Herz 2020:1-3. PMID: 32034445, doi: 10.1007/s00059-020-04909-z.

31. Lippi G, Plebani M. Laboratory abnormalities in patients with COVID-2019. infection. Clin Chem Lab Med. 2020. doi: 10.1515/cclm-2020-0198.

32. Tang N, Li D, Wang X, Sun Z. Abnormal Coagulation parameters are associated with poor prognosis in patients with novel coronavirus pneumonia. Journal of Thrombosis and Haemostasis 2020. doi: 10.1111/ jth.14768. 
33. He J, Wu B, et al. Characteristic ECG Manifestations in Patients with COVID-19. Canadian Journal of Cardiology 2020. doi: 10.1016/j.cjca.2020.03.028.

34. Castagne B, Viprey M, et al. Cardiovascular safety of tocilizumab: A systematic review and network metaanalysis. PloS one 2019;14(8). doi: 10.1371/journal. pone. 0220178 .

35. Tonnesmann E, Kandolf R, Lewalter T. Chloroquine cardiomyopathy - a review of the literature. Immunopharmacol Immunotoxicol 2013;35:434-42. doi: 10.3109/08923973.2013.780078.

36. Page RL, O'Bryant CL, Cheng D, et al. Drugs That May Cause or Exacerbate Heart Failure:A Scientific Statement From the American Heart Association. Circulation 2016;134:e32-69. doi: 10.1161/CIR.0000000 000000426.

37. Aljofan M, Gaipov A. COVID-19 Treatment: The Race Against Time. Electron J Gen Med. 2020;17(6):em227. doi: 10.29333/ejgm/7890.

38. Szerlip M, Anwaruddin S, et al. Considerations for Cardiac Catheterization Laboratory Procedures During the COVID-19 Pandemic Perspectives from the Society for Cardiovascular Angiography and Interventions Emerging Leader Mentorship (SCAI ELM) Members and Graduates. Catheterization and cardiovascular interventions: official journal of the Society for Cardiac Angiography \& Interventions 2020. doi: 10.1002/ ccd.28887.
39. Tam C-CF, Cheung K-S, et al. Impact of Coronavirus Disease 2019 (COVID-19) Outbreak on ST-SegmentElevation Myocardial Infarction Care in Hong Kong, China. Circulation: Cardiovascular Quality and Outcomes 2020. doi: 10.1161/CIRCOUTCOMES.120. 006631.

40. Wu Z, McGoogan JM. Characteristics of and important lessons from the coronavirus disease 2019 (COVID-19) outbreak in China: summary of a report of 72314 cases from the Chinese Center for Disease Control and Prevention. JAMA 2020. PMID: 32091533, doi: 10.1001/ jama.2020.2648.

41. Lazzerini M, Putoto G. COVID-19 in Italy: momentous decisions and many uncertainties. The Lancet Global Health 2020. doi: 10.1016/S2214-109X(20)30110-8.

42. World Health Organization/ Europe [Internet]. Strengthening the health system response to COVID-19. Recommendations for the WHO European Region;2020 March [Cited April 3]. Available at: http://www.euro. who.int/en/health-topics/health-emergencies/coronavir us-covid-19/novel-coronavirus-2019-ncov-technical-gu idance/coronavirus-disease-covid-19-outbreak-technic al-guidance-europe/strengthening-the-health-systemsresponse-to-covid-19

43. Peeri NC, Shrestha N, et al. The SARS, MERS and novel coronavirus (COVID-19) epidemics, the newest and biggest global health threats: what lessons have we learned?. International journal of epidemiology 2020. doi: 10.1093/ije/dyaa033. 